\title{
LA AUDITORÍA Y SU INFLUENCIA EN EL CONTROL DE LAS ACTIVIDADES REALIZADAS POR LAS EMPRESAS
}

\author{
THE AUDIT AND ITS INFLUENCE ON THE CONTROL OF THE ACTIVITIES CARRIED \\ OUT BY THE COMPANIES
}

Lady Diana Zambrano Montesdeoca Universidad Técnica de Manabí Manabí, Ecuador ORCID: https://orcid.org/0000-0002-8056-4804 Correo electrónico: ay_abao@hotmail.com

Cruz Nathaly Gilces Vidal Universidad Técnica de Manabí Manabí, Ecuador

ORCID: https://orcid.org/0000-0002-8955-846X Correo electrónico: nathy_0394@ @hotmail.com

\section{RESUMEN}

Objetivo: Conocer sobre la auditoría y su influencia en el control de las actividades realizadas por las empresas. Método: El enfoque del estudio fue cuantitativo, el tipo de investigación descriptiva, correlacional y exploratoria; el diseño transeccional no experimental. Se realizó una investigación de campo, con visitas a las empresas, empleando encuestas y conociendo su realidad; a su vez se recurrió a la investigación histórica, con la cual se obtuvo información auténtica de las empresas y de la utilización de auditorías a nivel empresarial. Resultados: Las auditorías influyen en el control de las actividades que realizan las empresas, ya que pueden ayudar a las entidades a mejorar su funcionalidad y alcanzar significativos ahorros en los costos. Conclusiones: Se logró determinar la situación actual de las empresas en estudio, conociendo sus fortalezas y debilidades, lo cual permitió conocer su operatividad, con el propósito de verificar la veracidad de las auditorías y justificar el uso de sus recursos de manera eficiente constatando la importancia de llevar a cabo este tipo de control minucioso y exhaustivo cada determinado tiempo en las organizaciones.

Palabras clave: Auditoría; contables; control; recursos; riesgos.

\begin{abstract}
Objective: To learn about the audit and its influence on the control of the activities carried out by the companies. Method: The focus of the study was quantitative, the type of research descriptive, correlational and exploratory; the non-experimental transectional design. A field investigation was carried out, with visits to companies, using surveys and learning about their reality; in turn, historical research was used, with which authentic information was obtained from the companies and the use of audits at the company level. Results: Audits influence the control of activities carried out by companies, as they can help entities improve their functionality and achieve significant cost savings. Conclusions: The present investigation managed to determine the current situation of the companies under study, knowing their strengths and weaknesses, which allowed us to know their operation, in order to verify the veracity of the audits in order to justify the use of their resources efficiently, confirming the importance of carrying out this type of meticulous and exhaustive control from time to time in organizations.
\end{abstract}

Keywords: Audit; accounting; control; resources; risks.

(c) Los autores. Este artículo es publicado por la Revista Quipukamayoc, Universidad Nacional Mayor de San Marcos. Este es un artículo de acceso abierto, distribuido bajo los términos de la Licencia Creative Commons Atribución-NoComercial-Compartirlgual 4.0 Internacional.(http://creativecommons.org/licenses/by-nc-sa/4.0/), que permite el uso no comercial, distribución y reproducción en cualquier medio, siempre que la obra original sea debidamente citadas. 


\section{INTRODUCCIÓN}

Los primeros indicios sobre la utilización del término auditor se encuentran en escritos atribuidos a Aristófanes, César y Cicerón en Grecia y Roma, de manera simultánea. Varios escritos mencionan que existen documentos con una antigüedad aproximada de 2500 años que revelan que durante el reinado de Ptolomeo Filadelfo II se practicaban auditorías internas para administrar el estado de Grecia; estas nacientes auditorías se ocupaban de examinar la exactitud de los registros contables y evaluaban que fueran apropiadas las actividades reflejadas en las cuentas (Santillana, 2013).

En la actualidad, las organizaciones a nivel mundial funcionan mediante procesos con la finalidad de controlar cada actividad de una empresa para que esta se desarrolle de una manera eficiente, por lo que es importante el control interno aplicado a los manuales de procedimientos, los que al mismo tiempo son guías operativas para el proceso que se asigna a una persona o actividad dentro de una organización (Vergara, 2017).

No obstante, otros autores mencionan que, en la década de los años 90, en los círculos de profesionales de la información de la academia mundial, comenzó a acrecentarse el interés por temas relacionados con la auditoría de la información. A partir de este momento, esta se empieza a promover como una novedad en las prácticas de la gestión de información (Martínez y Armenteros, 2006).

Las auditorías surgen de la necesidad de verificar las actividades que se han desarrollado dentro de una empresa y así llevar un control detallado de las operaciones desempeñadas. Sin embargo, Vergara (2017) menciona que las auditorías nacieron durante la revolución industrial, con el fin de reconocer las malas acciones que se establecieron dentro las organizaciones y, en consecuencia, su control interno asegura que la información contable sea confiable frente a los fraudes que se presenten, dando una eficiencia y eficacia operativa para ejecutar las actividades.

Otro autor, Sandoval (2012) describe que la auditoria:

Significa verificar que la información financiera, administrativa y operacional que se genera es confiable, veraz y oportuna, es revisar que los hechos, fenómenos y operaciones se den en la forma en que fueron planteados, que las políticas y procedimientos establecidos se han observado y respetado, es evaluar la forma en que se administra y opera para aprovechar al máximo los recursos (p. 9).

Asimismo, para los autores Arens, Elder, y Beasley (2007) la auditoría es "la acumulación y evaluación de la evidencia basada en información para determinar y reportar sobre el grado de correspondencia entre la información y los criterios establecidos, describiendo que puede realizarla una persona independiente y competente" (p. 4).

En relación con el sistema de control interno, este es fundamental para las entidades, ya que integra normas y procedimientos para el flujo de toda la información administrativa financiera con las distintas operaciones que realiza la entidad, de forma detallada, lo cual beneficia a la empresa, pues brinda una seguridad sobre la razonabilidad de sus estados financieros (Vergara, 2017).

Tal es así que Florian (2016) indica que, a medida que ha transcurrido el tiempo, el concepto de auditoría ha ido evolucionando hasta convertirse en un instrumento de mucha importancia para vigilar el proceso objetivo y veraz de las entidades y, de esta manera, optimizar sus recursos. Por ello, las empresas deben contar con los medios pertinentes para el registro controlado de sus actividades y que sean eficientes para alcanzar sus objetivos empresariales y productivos; de este modo, la auditoría se ve ligada a la detección de errores y fraudes dentro de los estados financieros contables y de la contabilidad que los produce, esta detección se realiza por medio de la revisión y comunicación de hallazgos o evidencias (Montilla y Herrera, 2006).

En el ámbito académico, Sosa (2015) reveló que la problemática del estudio realizada a la empresa Golden Amazon Group S.A.C es la deficiencia que presenta su área de gestión contable, ya que no considera el control interno como una alternativa de equilibrio para mejorar el ámbito financiero y organizacional. Según los resultados, se observa que la variable de control interno expresa una correlación del 0,865 , lo cual indica que existe una relación positiva de estudio, sobre una muestra de 13 individuos, con la variable de gestión contable, por consiguiente, se podría manifestar, efectivamente, que el control interno incide en la gestión contable de la empresa.

Cumpa (2018) concluyó que el 37\% de las actividades de auditoría de la empresa Rodson Music se logra cumplir; pero un $63 \%$ no, lo cual indica que la auditoría interna realizada a la empresa es deficiente. Asimismo, los resultados de la lista d-e cotejo muestran que el $27 \%$ de las actividades de control contable se logra cumplir, pero no el $73 \%$, esto evidenció el incumplimiento de las normas las cuales tuvieron que ser corregidas por la empresa. Por otro lado, se observa que la correlación obtenida entre la auditoría y las actividades de control es del 0,695, existe una relación significativa entre las variables de la investigación pese a que la empresa muestra carencias con respecto a la auditoría interna que perjudica el control contable. 
Chumpitazi y Salazar (2019), en su investigación, aplicaron la auditoría externa para obtener conocimientos del procesamiento de información; también realizaron la evaluación de los controles y sus posibles riesgos, para llegar a una conclusión sobre la importancia de la implementación de controles internos en el manejo de la información financiera.

Arana (2015) concluye que la auditoría financiera influyó positivamente en la gestión de las pequeñas empresas manufactureras del régimen general del impuesto a la renta, dado que es una técnica fundamental para el desarrollo y crecimiento de las empresas, además, otorga posibilidades de cambio y perfeccionamiento.

Diferente a lo que explican Serrano, Señalin, Vega y Herrera (2017), quienes en su investigación demuestran que es necesario cuidar las acciones que realiza la administración de las organizaciones, pues indican que cuando la información financiera posee el contenido e información correcta, esta les proporciona a los usuarios las herramientas indispensables para la toma de decisiones.

Conforme a lo mencionado, se destaca que el objetivo de las auditorías es comprobar de manera sistemática, veraz y oportuna el registro de los estados financieros, de acuerdo con las evidencias administrativas, contables y de gestión para su debido cumplimiento y desarrollo.

\section{MATERIALES Y MÉTODOS}

El tipo de investigación fue descriptiva, correlacional y exploratoria, siendo el diseño no experimental transeccional. Para este estudio, de una población de 50 empresas ubicadas en la ciudad de Portoviejo (Ecuador), para la selección de los propietarios de las empresas se utilizó la técnica del muestreo accidental o casual considerando 15 de ellas a las cuales se les aplicó una encuesta.

El enfoque del estudio para esta investigación fue de tipo cuantitativo con la finalidad de realizar la recolección de datos con base a la medición numérica y el análisis estadístico que permita conocer la situación de los procesos de auditoría en las empresas, por ello, se utilizaron otras técnicas tales como la investigación de campo, a través de visitas a las empresas; como también la investigación histórica, con la cual se obtuvo datos operativos y de la periodicidad de la realización de las auditorías.

Los datos estadísticos obtenidos fueron presentados en tablas dinámicas para su mejor interpretación, esto permitió conocer las fortalezas y debilidades de las organizaciones, considerando que muchas no llevan un control exhaustivo en la revisión de los procedimientos contables, administrativos, laborales, etc., que se llevan a cabo en procesos de auditoría.

\section{RESULTADOS}

De acuerdo con las variables de estudio, se logró determinar la incidencia de la auditoría en el control de las actividades realizadas por las empresas, dando como resultado que, del total de las empresas objeto de estudio, el 73,33\% sí realiza su examen especial de auditoría a diferencia de las restantes que no ejecutaron auditorías desde el inicio de sus funciones, lo que corresponde al 26,67\%. Por ende, se pudo identificar el conocimiento de los procedimientos de auditorías que pueden facilitar a las empresas para lograr un buen control financiero y de gestión.

Del mismo modo, en el estudio realizado se constató que el 33,33\% de las empresas realiza una auditoría de tipo financiera, según el tipo de actividad operativa que ejecuta, a fin conocer la realidad económica de la entidad, dando importancia a los demás tipos de auditorías como lo describe la figura 1.

De los resultados obtenidos de las empresas que realizan periódicamente las auditorías, el 33,33\% menciona que este procedimiento previene del fraude y de posibles riesgos originados por irregularidades o por actos inadecuados; el 26,67\% manifiesta que facilita tomar decisiones adecuadas; el $26,67 \%$ atribuye a la auditoría un aumento de confianza de los accionistas, ya que les permitió conocer la situación actual de la empresa; mientras que el 13,33\% considera que la auditoría sirve para ordenar y mejorar los procesos con el objetivo de manejar bien los recursos. En relación con el tiempo de periodicidad para realizar las auditorías, el $80 \%$ consideró la práctica anual de este proceso, a diferencia del $20 \%$ que prefiere realizarla cada 3 años debido a que no poseen tanto dinero para contratar personal que realice este examen, debido a la falta de recursos para contratar personal que realice este examen.

Referente al equipo auditor, el $66,67 \%$ prefiere que las auditorías sean realizadas por varios especialistas de diferentes áreas para que, de acuerdo con el tipo de empresa, el examen sea más detallado; el $20 \%$ refiere que una auditoría debe ser ejecutada por un auditor; mientras que el $13,33 \%$ indica que los encargados deben ser especialistas del área contable.

En relación con la utilidad de la aplicación de la auditoría, un 53,33\% de los encuestados manifiesta que sirve para el monitoreo del desempeño de actividades de la empresa, con el fin de conocer si se cumple con los objetivos planteado. Estos resultados se presentan en la figura 2.

La auditoría es un elemento importante para la toma de decisiones dentro de una empresa, ya que permite descubrir falencias, prevenir errores y realizar cambios que ayuden a la institución a superarse y a mantenerse en el mercado. 


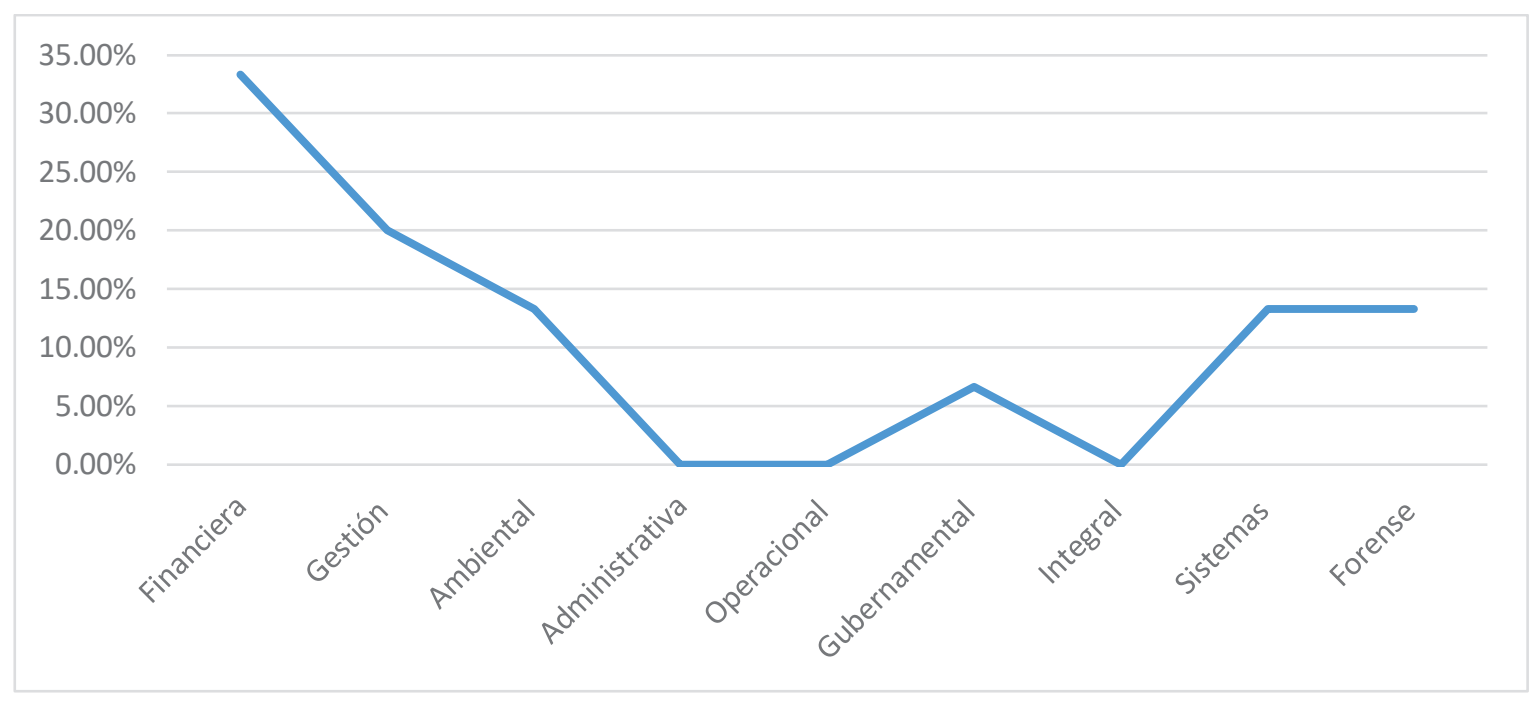

Figura 1. Tipos de auditorías.

Fuente: Elaboración propia a partir de encuestas realizadas.

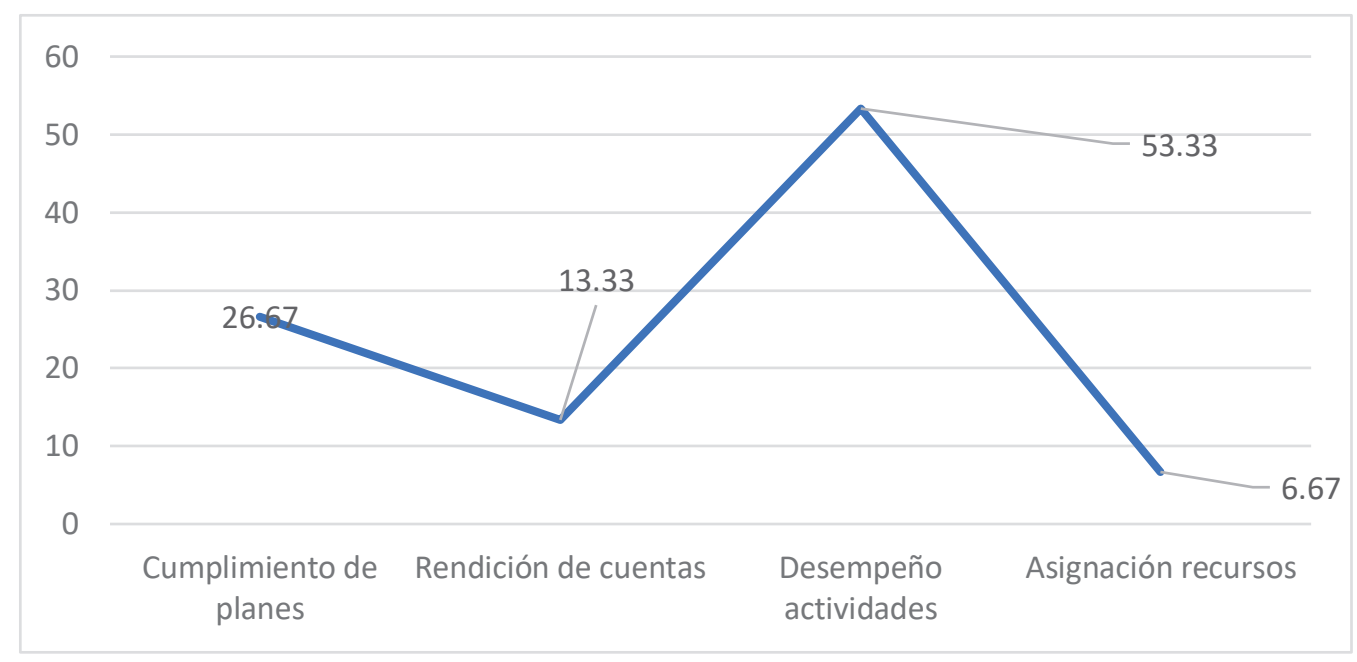

Figura 2. Utilidad de las auditorías.

Fuente: Elaboración propia a partir de encuestas realizadas.

\section{DISCUSIÓN}

De acuerdo con los datos obtenidos y según lo descrito en varias investigaciones acerca de este tema, se constata que la auditoría es un examen que permite conocer la situación de la empresa, es decir, sus estados financieros y cómo sus recursos son empleados para lograr las metas planteadas; para ello es importante destacar el tiempo de periodicidad de las auditorías y el cumplimiento de la normativa vigente. La presente investigación determinó la situación actual del objeto en estudio, a saber, la auditoría y su influencia en el control de las actividades de las empresas, pues se ha conocido su respectiva operatividad con el propósito de verificar la veracidad de las auditorías a fin de justificar el uso de los recursos de las empresas de manera eficiente. Se ha comprobado la importancia de llevar a cabo este tipo de control minucioso y exhaustivo cada cierto tiempo en las organizaciones, y se ha determinado la incidencia de la auditoría en el control de las actividades realizadas por las empresas. Estos resultados se comparten 
con Grimaldo (2014), quien menciona que las auditorías ofrecen la oportunidad de conocer en tiempo real el estado actual de las empresas, pues favorece la formulación de sugerencias para su mejoramiento; y con Hernández (2016), quien refiere que la base del buen funcionamiento de una organización está enmarcada en el entorno de control; sin embargo, muchas empresas desconocen su importancia para prevenir cualquier aspecto que pudiese influenciar de manera negativa a la empresa. Asimismo, con Serrano et al. (2017), ya que los resultados obtenidos en esta investigación evidencian que las empresas cumplen con la mayor parte de los componentes del control interno, garantizando que las actividades y operaciones avalen la legalidad y razonabilidad de la información que se genera.

\section{REFERENCIAS}

Arana, E. (2015). La auditoría financiera y su influencia en la gestión de las pequeñas empresas manufactureras del régimen general del Impuesto a la Renta en la ciudad de Tarapoto. Año 2014 (tesis para optar el título de contador público ). Recuperada de: http://repositorio.unsm.edu.pe/bitstream/handle/11458/1917/ ITEM\%4011458-651.pdf?sequence=1\&isAllowed=y

Arens, A. A., Elder, R. J., y Beasley, M. S. (2007). Auditoría. Un enfoque integral (13. ${ }^{\mathrm{a}}$ ed.). México D.F.: Pearson.

Chumpitazi, P. y Salazar, R. (2019). La influencia del control interno en la auditoría financiera en la empresa La Floresta S.A.C. por el periodo 2015 y 2016 (tesis para optar el título de contador público). Recuperada de: http://repositorio.utp.edu.pe/bitstream/UTP/2025/1/ Pierina\%20Chumpitazi_Rossana\%20Salazar_Trabajo\%20de\%20Suficiencia\%20Profesional_Titulo\%20 Profesional_2019.pdf

Cumpa, M. (2018). Auditoría interna y su influencia en el control contable en la Empresa Rodson Music, Chiclayo- 2018 (tesis para obtener el grado de bachiller en Contabilidad). Recuperada de http://repositorio.uss. edu.pe/bitstream/handle/uss/5249/Cumpa\%20Bello. pdf? sequence $=1$ \&isAllowed $=y$

Florian, C. (2016). La Auditoría, Origen y Evolucion. ¿Por qué en Colombia se conoce solo a través de leyes? Recuperado de: http://www.unilibre.edu.co/bogota/pdfs/2016/4sin/B20.pdf
Grimaldo, L. (2014). La importancia de las auditorías internas y externas dentro de las organizaciones (tesis para optar el título de contador público). Recuperada de: https://repository.unimilitar.edu.co/bitstream/handle/10654/13537/Importancia\%20de\%20las\%20Auditorias.pdf;jsessionid=2EB8C496A01A48826DB17CF0A28229DE? sequence $=1$

Hernández, O. (2016). La auditoría interna y su alcance ético empresaria. Actualidad Contable Faces, 19(33),15-41. Recuperado de: https://www.redalyc. org/pdf/257/25746579003.pdf

Martínez, M. y Armenteros, I. (2006). Orígenes y clasificación de la auditoría de la información. ACIMED, 14(5). Recuperado de: http://scielo.sld.cu/scielo.php?scrip$\mathrm{t}=$ sci_arttext\&pid=S1024-94352006000500017\&ln$\mathrm{g}=\mathrm{es} \& \mathrm{t} \operatorname{lng}=\mathrm{es}$.

Montilla, O. y Herrera, L. (2006). El deber ser de la auditoría. Estudios Gerenciales, (98), 83-110. Recuperado de: https://www.icesi.edu.co/revistas/index.php/estudios_gerenciales/article/view/185/183

Sandoval, H. (2012). Introducción a la Auditoria. México D.F.: Red Tercer Milenio.

Santillana, J. R. (2013). Auditoría Interna (3. a ed.). México D.F.: Pearson.

Serrano, P., Señalin, L., Vega, F. y Herrera, P. (2017). El control interno como herramienta indispensable para una gestión financiera y contable eficiente en las empresas bananeras del cantón Machala (Ecuador). Espacios, 39(3), 30. Recuperado de: https://www.revistaespacios.com/a18v39n03/a18v39n03p30.pdf

Sosa, L. (2015). El control interno y su influencia en la gestión contable de la empresa Golden Amazon Group S.A.C. (tesis para optar el título de contador público). Recuperada de http://repositorio.autonoma.edu.pe/ bitstream/AUTONOMA/152/1/SOSA\%20PEREZ.pdf

Vergara, I. M. (2017). Los manuales de procedimientos como herramientas de control interno de una organización. Revista Universidad y Sociedad, 9(3), 247-252. Recuperado de: https://rus.ucf.edu.cu/index.php/rus/ article/view/637 
